\title{
Siblings of opposite sex as a risk factor for child mortality
}

\author{
Peter Aaby, Kåre Mølbak
}

\begin{abstract}
Objective-To examine whether contracting an infection from a sibling of the opposite sex affects child mortality.

Design-Retrospective study of twins and case-control study of randomly selected children conducted within a prospective cohort study.

Setting-Urban and rural areas of Guinea-Bissau.

Subjects-251 Pairs of twins (65 male pairs, 72 female pairs, and 114 mixed pairs), and 39 children who died and 78 controls identified in a cohort of 853 children aged less than 4 , comprising 772 randomly selected children, all twins, and all children who had measles living in Bandim 2.

Main outcome measures-Death of a child and sex of cotwin or older sibling nearest in age.

Results-The mortality among children from mixed pairs of twins was higher than that among pairs of the same sex (relative risk 1.47, 95\% confidence interval $1 \cdot 14$ to 1.90 ). Boys had a slightly higher mortality especially in the older groups. In the case-control study the older sibling nearest in age was of the opposite sex for $29(74 \%)$ of the children compared with $36(46 \%)$ of the controls matched for age, sex, and area. Mortality was higher among both boys and girls with older siblings of the opposite sex than among those with siblings of the same sex (odds ratio 1.90, 0.78 to $4.61 v 7.5,1.59$ to $35 \cdot 30$ ), but the difference was more pronounced among girls.

Conclusions-Children whose older sibling nearest in age is of the opposite sex or who have cotwins of the opposite sex have a higher mortality from infectious diseases. Further investigation is needed to determine the nature of this relation.
\end{abstract}

\section{Introduction}

Studies of epidemics of measles in urban and rural areas in Guinea-Bissau have consistently found a higher mortality when the infection was contracted from someone of the opposite sex. ${ }^{1}$ Reports of fatal measles in medical publications indicate a similar tendency.' As this suggests a previously unrecognised mechanism for severe infection we examined whether contracting a disease from someone of the opposite sex compared with someone of the same sex increased child mortality in general. In two studies of twins in Guinea-Bissau we examined whether mixed pairs of twins had a higher mortality than pairs of the same sex. In addition, we report a case-control study examining whether the sex of the older sibling nearest in age affected child mortality.

Statens Seruminstitut, Copenhagen, Denmark Kåre Mølbak, $\mathrm{MD}$, research fellow

Correspondence to: Dr Aaby.
Subjects and methods REGISTRATION OF TWINS

Censuses were carried out in 1987 and 1988 in Bandim 1 and 2, two districts with a population of roughly 20000 in the capital of Guinea-Bissau. Information on twin state, age, sex, and cause of death, where applicable, for each cotwin was obtained for people of all ages. The identity and area of residence of surviving cotwins were controlled so that surviving pairs of twins living in the same area were not counted twice. For younger twins, information obtained in the census was compared with data from the prospective birth register in the two areas. The data from the two sources corresponded well: 40 pairs of twins were identified in the census and 40 pairs should have been identified in the birth register, but two children born on the same day to different mothers living in the same household had been erroneously registered as twins and one mother had two sets of twins according to the birth register but only one set according to the census.

A census with the same questions about twin state was carried out in five villages in the rural area of Quinhamel in 1988. The people in Quinhamel, who have been followed up since $1978,{ }^{2}$ all belong to the Pepel tribe, the same ethnic group that predominates in Bandim ( $60 \%$ of the population).

\section{CALCULATION OF MORTALITY}

Mortality in a particular age group was calculated when one twin had died, provided that the cotwin had survived to the beginning of the relevant age group, and was expressed as a proportion of the number of pairs of twins in which one cotwin or both twins had lived through the whole age group. For example, a pair in which the surviving twin was 14 years of age at the time of the census and the sibling had died at the age of 6 years was counted as one surviving pair for the intervals $<1$ month and 1-59 months and as one death in the interval 5-9 years (see table I). After controlling that hazards were proportional a Cox's regression model for grouped data ${ }^{3}$ was used to calculate the mortality hazards ratio with the generalised linear interactive modelling (GLIM) statistical package.

\section{CASE-CONTROL STUDY}

Children aged under 4 living in 301 randomly selected houses in Bandim 2 were studied from April 1987 to March 1988 by weekly questionnaires on morbidity. All twins and children who had measles living in Bandim 2 and babies born in the 301 houses during the study period were also included. Those children who were sick when visited by an assistant were examined by a doctor. The mothers of the children who died were interviewed to obtain information on the cause of death and the size and sexes of the family at the time of the child's illness. For each dead child, two controls were selected from the surviving children in the study. Controls were matched for sex, subarea, and month of birth (within two months). Composition of the family was normally classified according to the sex of the older sibling nearest in age with the same mother. For $10(26 \%)$ of the children who died and $19(24 \%)$ of the controls 
classification was based on the sex of the next oldest household member, usually a half sibling because there was no older sibling with the same mother. The children who died and their two control children were compared with the Mantel-Haenszel estimator of the odds ratio. ${ }^{4}$

\section{Results}

MORTALITY AMONG TWINS

Among the 251 pairs of twins with at least one surviving twin registered in the urban districts the distribution was male pairs $26 \%$ (65), female pairs $29 \%$ (72), and mixed pairs $45 \%$ (114) (table I). This was similar to the outcome among 171 prospectively registered women pregnant with twins in the same urban areas: male pairs $29 \%$ (49), female pairs $28 \%$ (48), and mixed pairs $43 \%$ (74). Among the 251 pairs of twins identified in the urban census one of the pair had died in $42 \%(27 / 65)$ of male pairs and $38 \%$ (27/72) of female pairs compared with $58 \%(66 / 114)$ of mixed pairs (relative risk $=1 \cdot 47,95 \%$ confidence interval $1 \cdot 14$ to $1.90 ; \mathrm{p}<0 \cdot 01)$. Even when the seven children who had died from measles were excluded the mortality in mixed pairs was significantly higher than that in pairs of the same sex $(1.48,1 \cdot 13$ to 1.94$)$.

Boys had a slightly higher mortality than girls, particularly in the older age groups (table I). Analysis with Cox's regression model for grouped data and standardising for sex gave a postneonatal mortality hazards ratio of 2.12 for mixed pairs compared with same sex pairs $(95 \%$ confidence interval 1.27 to $3 \cdot 54$; $\mathrm{p}<0.01)$. In this analysis the postneonatal mortality ratio was 1.67 for boys compared with girls $(1.00$ to $2 \cdot 78)$.

TABLE I - Mortality at various ages among twins according to sex of the pair

\begin{tabular}{lccc}
\hline & \multicolumn{3}{c}{ No of deaths/No of pairs (\%) } \\
\cline { 2 - 4 } Age & Male pairs & Female pairs & Mixed pairs \\
\hline <1 Month & $7 / 65(11)$ & $8 / 72(11)$ & $8 \mathrm{M}+7 \mathrm{~F} / 114(13)$ \\
1-59 Months & $9 / 39(23)$ & $11 / 47(23)$ & $20 \mathrm{M}+14 \mathrm{~F} / 72(47)$ \\
5-9 Years & $3 / 20(15)$ & $0 / 28$ & $3 \mathrm{M} / 24(13)$ \\
10-14 Years & $1 / 11(9)$ & $0 / 21$ & $1 \mathrm{M} / 15(7)$ \\
\hline
\end{tabular}

The results for the 31 pairs of twins identified in the census in the villages of Quinhamel gave similar results. The mortality was $57 \%(8 / 14)$ in mixed pairs and $29 \%(5 / 17)$ in same sex pairs (relative risk $=1.94$, 0.83 to 4.53 ). The relative risk for postneonatal mortality was $3 \cdot 5(1 \cdot 15$ to $10 \cdot 67)$.

\section{CASE-CONTROL STUDY}

Of the 853 children in the study, 66 died: 15 were stillborn or died aged $<1$ month and one premature baby died aged 39 days. The remaining 50 died aged between 4 and 34 months, but information was not available about the cause of death and size of family for two children. Of the 48 children for whom information was available, 39 had at least one older sibling living in the same house (table II). The older sibling nearest in age was of the opposite sex for $74 \%$ (29) of the

TABLE II -Sex of older siblings nearest in age to children who died and of control children

\begin{tabular}{lrrrrrr}
\hline & \multicolumn{3}{c}{ Children who died } & & \multicolumn{2}{c}{ Control children } \\
\cline { 2 - 3 } \cline { 6 - 7 } Older sibling nearest in age & M & F & & M & F \\
\hline M & 8 & 14 & & 25 & 15 \\
F & 5 & 2 & & 21 & 17 \\
No older sibling & 5 & 4 & & & \\
\hline Total & 28 & 20 & & 46 & 32 \\
\hline
\end{tabular}

39 children who died and $46 \%$ (36) of the control children (odds ratio $=2 \cdot 83,95 \%$ confidence interval 1.24 to 6.48$)$. Mortality was higher for both boys ( 1.90 , 0.78 to $4 \cdot 61)$ and girls $(7 \cdot 50,1 \cdot 59$ to $35 \cdot 30)$ whose older sibling nearest in age was of the opposite sex, though the difference was most pronounced for girls. Even when the four children who died from measles were excluded the odds ratio was still significantly higher for children whose older sibling nearest in age was of the opposite $\operatorname{sex}(2 \cdot 50,1 \cdot 16$ to $5 \cdot 39)$. In seven families with only two children six children who died had siblings of the opposite sex but only one had a sibling of the same $\operatorname{sex}(\mathrm{p}=0 \cdot 06$, one side binomial test).

The pattern of mortality seemed to vary by cause of death. All children who died from measles (4/4) and nearly all who died from acute diarrhoea $(7 / 8)(7 \cdot 00$, $0 \cdot 78$ to $63 \cdot 10)$ and chronic diarrhoea $(11 / 12)(7 \cdot 50,1 \cdot 80$ to $31 \cdot 18)$ had next eldest siblings of the opposite sex. Among children who died from pneumonia $(2 / 6)$ and other causes (5/9), however, no similar tendency was found.

\section{Discussion}

Our study focused on postneonatal mortality because this is more likely to be related to infections transmitted by siblings than perinatal and neonatal mortality. Both the study of twins and the case-control study suggested that when the older sibling nearest in age is of the opposite sex this is an important risk factor for postneonatal death. All the children apparently died of infectious diseases. As the slight excess in mortality among boys was found both for male pairs compared with female pairs and between boys and girls in mixed pairs it seems unlikely that differential care plays an important part in the higher mortality in mixed pairs.

The data in table I probably underrepresent the risk of death among twins as the study did not detect pairs in which both twins had died. There was probably some underreporting of pairs of twins in the census, particularly among pairs in which one had died. The tendency towards higher mortality among mixed pairs, however, is unlikely to have been caused by misreporting related to sex, especially as the relative prevalence of pairs of twins of the same sex in both Bandim (55\%) and Quinhamel (55\%) was what would be predicted from a rate of twins of one pair per 35-40 births (unpublished observations), the rate found in Bissau. Furthermore, the prevalence corresponded closely with the proportion of same sex twins born to women whose pregnancies were prospectively registered. A higher risk of both twins dying among same sex pairs compared with mixed pairs could also account for our results, but this would be associated with a reduction in the percentage of same sex pairs among older twins. If anything double deaths occurred more often in mixed pairs as of the 72 twins aged more than $10,48(67 \%)$ were same sex pairs (table I).

Thus the higher mortality among mixed pairs is probably due not to a higher risk of double deaths among same sex pairs but to a higher risk of postneonatal death in mixed pairs than in same sex pairs. This conclusion is strengthened by the fact that when the older sibling nearest in age was of the opposite sex this was an important risk factor for postneonatal mortality. This remained true when children who died from measles were excluded in both the study of twins and the case-control study. Thus transmission of infection to someone of the opposite sex may in general aggravate disease, as we have previously suggested for measles infection.' Further studies are needed to determine whether the underlying mechanism is biological or behavioural and whether it is a general 
phenomenon of infectious diseases or limited to certain infections such as measles.'

We thank Kim Knudsen for help with the statistical analysis. The study was supported by the Danish Council for Development Research, Danish Medical Research Council, Danish Social Science Research Council, University of Copenhagen, and European Community contract TS2-0179.
1 Aaby P, Bukh J, Hoff G, Lisse IM, Smits AJ. Cross-sex transmission of infection and increased mortality due to measles. Rev Infect Dis 1986;8 138-43.

2 Aaby P, Bukh J, Lisse IM, et al. Determinants of measles mortality in a rural area of Guinea-Bissau: crowding, age, and malnutrition. I Trop Pediatr 1984;30:164-9.

3 Kalbfleisch JD, Prentice RL. Marginal likelihoods based on Cox's regression and life model. Biometrika 1973;60:267-78.

4 Rothman KJ. Modern epidemiology. Boston: Little, Brown, 1986.

(Accepted 15 May 1990)

\title{
Short term linear growth in asthmatic children during treatment with prednisolone
}

\author{
Ole D Wolthers, Søren Pedersen
}

\begin{abstract}
Objective-To see whether small daily doses of prednisolone have any adverse effect on short term linear growth in children with mild asthma.

Design-Double blind, random order crossover trial of two dosages of prednisolone. During run in and washout periods patients were given placebo. All treatment periods were of two weeks' duration.

Setting-Outpatient clinic referrals in a secondary referral centre.

Patients - 14 Children (10 boys) aged 7-11 years with normal growth velocity during the previous year, no signs of puberty, and no history of receiving systemic or topical steroids during the two months before the study. One child was excluded because his pulmonary function deteriorated and another
\end{abstract} was withdrawn because of varicella.

Interventions-2.5 And $5.0 \mathrm{mg}$ prednisolone daily given in divided dosage in the morning and evening.

Main outcome measure-Growth of the lower leg as measured twice a week by knemometry.

Results-A significant reduction in mean growth velocity of the lower leg occurred in both prednisolone treatment periods. The mean difference between the run in period and the treatment period with $2.5 \mathrm{mg}$ prednisolone daily was $0.63 \mathrm{~mm} /$ week ( $95 \%$ confidence interval 0.47 to $0.80 \mathrm{~mm} /$ week) and between the run in period and the treatment period with $5.0 \mathrm{mg}$ prednisolone daily $0.57 \mathrm{~mm} /$ week $(0.38$ to $0.77 \mathrm{~mm} /$ week).

Conclusion-Small daily doses of prednisolone suppress short term linear growth in children with mild asthma. The clinical relevance of this finding needs further study.

\section{Introduction}

Growth suppression is a well known risk of long term treatment of children with oral steroids. ${ }^{12}$ For many reasons it has not been possible to study this side effect under controlled conditions, so little is known about the actual doses that cause growth suppression. During recent years, however, knemometry has become established as a powerful tool for monitoring short term linear growth. The knemometer measures changes in the length of the lower leg with a high reproducibility and an accuracy of $0.09-0 \cdot 16 \mathrm{~mm} .^{3.6}$ The method has been shown to be useful in evaluating the response to treatment with growth promoting substances, ${ }^{7-10}$ but only recently has it been suggested that it may also be used to detect growth suppression. ${ }^{112}$ We decided to carry out a study under controlled conditions and with the use of knemometry to see whether 2.5 and $5.0 \mathrm{mg}$ prednisolone daily had any adverse effect on short term linear growth in children with mild asthma.

\section{Patients and methods}

When planning the study we estimated the standard deviation of the mean growth rate of the lower leg to be $0.20 \mathrm{~mm} /$ week. ${ }^{9}{ }^{13}$ Given this assumption, we calculated that 12 patients would be enough to achieve a power of $>0.90$ in detecting a $50 \%$ reduction in growth rate, which was considered clinically relevant. ${ }^{14} \mathrm{~A} 15 \%$ rate of withdrawal was estimated, so necessitating increasing the study population required by two patients.

Ten boys and four girls aged 7-11 years (mean 9.1) entered the study. All had mild asthma, needing only treatment as required with inhaled $\beta_{2}$ stimulants. None had received inhaled or oral corticosteroids within two months before the study, and no other drugs were taken during the study period. All children were preadolescent without any signs of puberty. Their growth velocities during the previous year ranged from 3.0 to $9.0 \mathrm{~cm}$ (mean $5.1 \mathrm{~cm} /$ year). Height at the beginning of the trial varied from $-1 \cdot 19$ to $2 \cdot 72$ standard deviation scores (mean $0 \cdot 34$ ) and weight from 23.0 to $35.6 \mathrm{~kg}$ (mean $31.1 \mathrm{~kg}$ ). The body surface area of the children ranged from 0.97 to $1 \cdot 20 \mathrm{~m}^{2}$ (mean $1 \cdot 11$ $\mathrm{m}^{2}$ ). The study was approved by the local ethical committee and informed consent given by all children and their parents.

\section{STUDY DESIGN}

The study was a randomised double blind crossover trial with five periods of two weeks. In period 1 (run in) and periods 3 and 5 (washout) placebo was given, and in periods 2 and 4 the children took 2.5 and $5.0 \mathrm{mg}$ prednisolone daily. Treatment order was allocated by means of a computerised randomisation scheme prepared in balanced blocks. All medicine was divided into two daily doses and taken in the morning and evening. The tablets, identical in size and appearance, were delivered in identical glasses labelled with case number, period number, and prescription. Tablets were counted before and after each treatment period.

\section{MEASUREMENTS}

Knemometry of the right lower leg was scheduled for twice a week, a knemometer manufactured by the inventor being used. ${ }^{3}$ All measurements were performed by the same trained observer with no reference to previous days' recordings. The children were measured at roughly the same time (that is, within 30 minutes) in the afternoon (between 1 and $5 \mathrm{pm}$ ), as recommended for knemometry. ${ }^{6}$ At each visit four estimations of length of the lower leg were made, the mean of the last three measurements being used for 